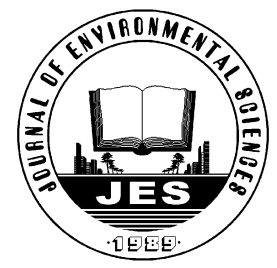

\title{
Adsorption of atrazine by natural organic matter and surfactant dispersed carbon nanotubes
}

\author{
Baoyou Shi*, Xiaoyan Zhuang, Xiaomin Yan, Jiajuan Lu, Hongxiao Tang \\ State Key Laboratory of Environmental Aquatic Chemistry, Research Center for Eco-Environmental Sciences, \\ Chinese Academy of Sciences, Beijing 100085, China. E-mail: byshi@rcees.ac.cn
}

Received 10 October 2009; revised 18 December 2009; accepted 21 December 2009

\begin{abstract}
The aggregation and dispersion behaviors of carbon nanotubes (CNTs) can regulate the environmental spread and fate of CNTs, as well as the organic pollutants adsorbed onto them. In this study, multi-walled carbon nanotubes (MWNTs) and single-walled carbon nanotubes (SWNTs) were surface modified with humic acids from different sources and with surfactants of different ionic types. The dispersion stability of surface modified CNTs was observed by UV-Vis spectrophotometry. The effect of humic acid and surfactant dispersion on the adsorption of atrazine by CNTs was investigated by batch equilibrium experiments. Both humic acid and surfactant could effectively disperse MWNTs, but not SWNTs, into stable suspensions under the studied conditions. Surface modified CNTs had a greatly reduced capacity for adsorption of atrazine. The inhibitory effect of peat humic acid was relatively stronger than that of soil humic acid, but the two surfactants had a similar inhibitory effect on atrazine adsorption by the two CNT types. Increases in surfactant concentration resulted in rapid decreases in the adsorption of atrazine by CNTs when the surfactant concentration was less than 0.5 critical micelle concentration.
\end{abstract}

Key words: carbon nanotubes; adsorption; dispersion; atrazine

DOI: $10.1016 / \mathrm{S} 1001-0742(09) 60238-2$

\section{Introduction}

Carbon nanotubes (CNTs) have unique structures and outstanding physical, chemical, and electronic properties and are widely used in areas such as catalysis, optical instrumentation, quantum computers, and the biochemical and medical industries. CNTs are also showing promise as potential solutions for environmental pollution challenges (Mauter and Elimelech, 2008). CNTs may be utilized to improve technologies for environmental sensing, pollutant detection, and for renewable energy. For example, many studies are now focused on the adsorption properties of CNTs (Chen et al., 2007; Yang et al., 2006) and their potential application as adsorbents for removal of pollutants from water (Kuo et al., 2008; Peng et al., 2003; Pan et al., 2008). However, the widespread application and requirements for large scale manufacture of these carbonaceous nanomaterials will inevitably lead to their release into the environment. The overall impacts of increased exposure to engineered nanomaterials on human health or the environment are not known, but are currently the focus of some studies (Colvin, 2003; Jia et al., 2005; Robichaud et al., 2005). CNT exposure has been reported to cause toxicological effects under certain circumstances (Monteiro-Riviere and Inman, 2006; Magrez et al., 2006).

\footnotetext{
* Corresponding author. E-mail: byshi@ rcees.ac.cn
}

CNTs are basically pure carbonaceous materials with high length/diameter aspect ratios. CNTs usually exist in the form of bundles and can not be dispersed in water due to the strong mutual attractive forces exerted by each tube and to the high hydrophobicity of their surfaces. Even intense mechanic or ultrasonic agitation is unable to disperse CNTs to form stable suspensions. However, many applications require CNTs to be separated into individual tubes, making CNT dispersion an important research direction (Marsh et al., 2007; Vaisman et al., 2006; Jiang et al., 2003).

Chemical modification (surface functionalization) of CNT surfaces, which can introduce electrical charge repelling forces between each tube, is one way to increase CNT dispersivity in water. Surfactant modification has become a frequently used approach for increasing CNT dispersion in water (Jiang et al., 2003; Lisunova et al., 2006). Yu et al. (2007) reported that multi-walled CNTs could be stably dispersed by surfactant (sodium dodecyl sulfonate) with sonication as the driving force. Natural organic matter (NOM), such as humic substances, has also been found effective for dispersion and stabilization of CNTs in aqueous phases (Hyung et al., 2007).

The dispersion stability of CNTs has important impacts on the environmental spread, the fate of CNTs themselves, and the pollutants adsorbed onto them. The improved 
dispersion of CNTs by surfactant or NOM treatment is based on surface modification, which in turn affects CNT capacity for adsorption of organic pollutants. In general, a NOM coating will significantly enhance the adsorption capacity of particulate adsorbents, such as natural mineral particles for hydrophobic organic compounds (Murphy et al., 1992). In contrast, for very fine materials such as activated carbon, NOM treatment can cause competitive and pore blockage effects that decrease the adsorption of organic pollutants (Li et al., 2002).

Wang et al. (2008) found that the pre-coating of CNTs with some organic materials could change their adsorption capacities for several organic contaminants, although the influence depended on the types of pre-coating materials used and the nature of the contaminants. However, to date, little attention has been paid to the effect of CNT dispersion on adsorption behavior. In contrast to natural particles and common carbon materials (such as activated carbon), CNTs usually exist in large aggregates (or bundles) and can change their aggregation status in response to different physical and chemical conditions. Dispersion of CNTs can increase adsorption sites to some extent, but dispersion reagents could also change the surface properties of CNTs. Thus, studies on the adsorption behavioral changes of CNTs before and after dispersion through surface modification are greatly needed (Pan and Xing, 2008).

In the present study, one type of multiple walled carbon nanotubes (MWNTs) and one type of single walled carbon nanotubes (SWNTs) were selected to evaluate the adsorption changes before and after dispersion by surfactants and by NOM. Both cationic and anionic surfactants and humic acids with different properties were used as surface modification reagents. Atrazine, one of the most frequently used triazine herbicides, was chosen as the model organic pollutant for conducting the adsorption experiments. The ubiquity and persistency of atrazine in the environment, and its risk as a recognized endocrine disruptor compound, make atrazine a target pollutant for water treatment studies (Chingombe et al., 2006). Many countries have set stringent regulations for atrazine levels in drinking water. In a previous study, we found that CNTs had a high capacity for atrazine adsorption and this adsorption was reversible (Yan et al., 2008). The main objective of the present study was to investigate the effect of CNT dispersion, induced by surfactant and NOM treatment, on the adsorption of atrazine. The dispersion stability of CNTs by humic acid and surfactant treatment was also evaluated.

\section{Materials and methods}

\subsection{Adsorbents}

SWNTs and MWNTs were purchased from Shenzhen Nanotech Port Technology Co. (Guangdong, China). Based on the information provided by the manufacturer, the diameters of SWNTs and MWNTs were $\leqslant 2 \mathrm{~nm}$ and 20-40 nm, respectively. The CNTs were used as received without further treatment. The elemental composition of SWNTs and MWNTs was analyzed using a MT-5 elemen- tal analyzer (Yanaco Chn Corder, Japan); the surface area and pore distribution were obtained with a BET surface area and pore size analyzer (QuadraSorb SI, USA).

\subsection{CNT dispersion reagents}

Two widely used surfactants, sodium dodecylbenzene sulfonate (SDBS) and cetyltrimethylammonium bromide (CTAB), were obtained from Beijing Chemical Reagent Company (China). SDBS (MW 348) is an anionic surfactant with a critical micelle concentration (CMC) of $433.5 \mathrm{mg} / \mathrm{L}$; CTAB (MW 364) is a cationic surfactant with a CMC of $361.0 \mathrm{mg} / \mathrm{L}$. A peat humic acid (PHA) was purchased from Tianjin Fine Chemicals Research Institute (China). Soil humic acid (denoted as SHA) was extracted from marsh soils (northwest area of Beijing), based on the method described by Salloum et al. (2001). To obtain the structural information on PHA and SHA, the elemental compositions of both were analyzed with a Vario EL Elemental Analyzer (Germany) and ${ }^{1} \mathrm{H}$ NMR was performed using a Varian Unityinova 500 Nuclear Magnetic Resonance Instrument (USA). Operation procedures of ${ }^{1} \mathrm{H}$ NMR were as previously described (Lu et al., 2009).

\subsection{Adsorbate}

Atrazine (2-chloro-4-ethylamino-6-isopropylamino1,3,5-triazine) with $97.7 \%$ purity was purchased from Wuxi Ruize Pesticide Co. (Jiangsu, China). Methanol used in this study was HPLC grade from Fisher Scientific Company (USA). All other reagents were analytical grade.

\subsection{Surface modification and dispersion of CNTs}

Humic acid stock solutions were prepared by dissolving $2.0 \mathrm{~g}$ of dry PHA or SHA into $1.0 \mathrm{~L} \mathrm{NaOH}(0.01 \mathrm{~mol} / \mathrm{L})$ by intense magnetic stirring for $24 \mathrm{hr}$. Non-dissolvable humic substances were separated by centrifugation. The background solution also contained $0.01 \mathrm{~mol} / \mathrm{L} \mathrm{NaNO}_{3}$ for ionic strength adjustment and $100 \mathrm{mg} / \mathrm{L} \mathrm{NaN}_{3}$ for bacterial growth inhibition. To prepare humic acid modified CNTs, $1.5 \mathrm{~g}$ of SWNTs (or MWNTs) were put into an Erlenmeyer flask, and $300 \mathrm{~mL}$ of humic acid stock solution was added. The flask was sealed and shaken for three days on an automatic shaker. The modified CNTs were then separated by filtration and rinsed several times using background solution to remove any loosely attached humic acids. The modified CNTs were freeze-dried for $48 \mathrm{hr}$, and then stored in a brown bottle for later use.

Similar procedures were used for preparing surfactantdispersed CNTs, replacing the humic acid stock solutions with 1.5 CMC SDBS or CTAB surfactant solutions.

\subsection{Dispersion stability of CNTs}

To observe the dispersion stability of CNTs treated by surfactants and humic acids, predetermined amounts of treated CNTs were transferred to glass tubes and diluted with an appropriate background solution. The amount of treated CNTs and the volume of solution were equivalent to that used in adsorption experiments. The glass tubes were agitated by ultrasonic treatment for $1 \mathrm{~min}$, and then allowed to settle. Samples were taken at different time 
intervals for absorbance measurement at $600 \mathrm{~nm}$ using a UV-Vis 8000 spectrophotometer (Shanghai Tianmei, China). Since the concentration of the original solution was too high and not suitable for absorbance measurement, it was diluted (50:1) before absorbance measurement.

\subsection{TEM observation}

Images of CNTs dispersed by humic acids and surfactants were obtained by transmission electron microscopy (TEM H7500, Hitachi, Japan). Samples were prepared by placing droplets of aqueous CNT suspensions onto coppercarbon grid sample holders and dried overnight at room temperature.

\subsection{Adsorption experiments}

Adsorption experiments were conducted using batch reaction procedures. CNT adsorbents $(20 \mathrm{mg})$ were transferred to $40 \mathrm{~mL}$ glass centrifuge tubes and $20 \mathrm{~mL}$ of atrazine solution (in background solution of $0.01 \mathrm{~mol} / \mathrm{L}$ $\mathrm{NaNO}_{3}$ and $100 \mathrm{mg} / \mathrm{L} \mathrm{NaN}$ ) was added at a predetermined concentration. The tubes were tightly sealed with Teflon-lined screw caps and shaken on a reciprocating shaker for $24 \mathrm{hr}$ at $(25 \pm 1)^{\circ} \mathrm{C}$. Preliminary kinetic tests indicated that adsorption equilibrium could be reached within $24 \mathrm{hr}$ (Zhuang et al., 2009). After a $24 \mathrm{hr}$ equilibration, CNTs were separated by filtration and the residual atrazine concentration in the solution was analyzed by high performance liquid chromatography HPLC (1525, Waters, USA). The operating conditions were: mobile phase $85 / 15(V / V)$ of methanol/water, flow rate $1 \mathrm{~mL} / \mathrm{min}$, $\mathrm{C} 18$ column temperature $35^{\circ} \mathrm{C}$, detection wavelength 223 $\mathrm{nm}$. The retention time of atrazine was around $4.2 \mathrm{~min}$. For each adsorption experiment blanks without CNTs were run in parallel to assess other possible losses of atrazine during the adsorption experiments. The adsorption experiments and blanks were all run in duplicates. The amount of adsorbed atrazine was calculated from the atrazine concentration differences between the solutions after adsorption and the blanks. The atrazine loss during adsorption process and filtration was negligible. Atrazine adsorbed was calculated by the following Eq. (1):

$Q=\frac{\left(C_{0}-C_{\mathrm{e}}\right) V}{m}$

where, $Q(\mathrm{mg} / \mathrm{g})$ is the amount of atrazine adsorbed by adsorbent; $C_{0}(\mathrm{mg} / \mathrm{L})$ and $C_{\mathrm{e}}(\mathrm{mg} / \mathrm{L})$ are the initial atrazine concentration and the equilibrium aqueous concentration, respectively. $V(\mathrm{~mL})$ is the solution volume; $m(\mathrm{mg})$ is the mass of adsorbents. The Freundlich model was adopted to fit the adsorption isotherm:

$Q=K_{\mathrm{f}} C_{\mathrm{e}}^{n}$

where, $Q(\mathrm{mg} / \mathrm{g})$ is the adsorbate concentration in solid phase at equilibrium; $C_{\mathrm{e}}(\mathrm{mg} / \mathrm{L})$ is the adsorbate concentration in solution at equilibrium, $K_{\mathrm{f}}$ is the adsorption coefficient; $n$ is Freundlich equation exponent, an indicator of the adsorption isotherm nonlinearity.

\subsection{Competitive adsorption of atrazine and surfactant on CNTs}

As surfactant concentration in solution is increased to the $\mathrm{CMC}$, the formation of surfactant micelles is gradually induced. Surfactant dispersion treatment was conducted using a relatively higher surfactant concentration (1.5 CMC). In order to understand the effect of changes in surfactant concentration on the adsorption of atrazine by CNTs, experiments of simultaneous competitive adsorption of surfactant and atrazine by non-dispersed CNTs were conducted. While holding atrazine concentration constant, the surfactant concentration was raised stepwise in the range of 0 to $1.0 \mathrm{CMC}$. The experimental procedures were as follows: a predetermined amount of CNTs was weighed into glass centrifuge tubes, and $10 \mathrm{~mL}$ of surfactant solution at selected concentration was added, followed by $10 \mathrm{~mL}$ of atrazine solution. Other procedures and analytical methods were as described above.

\section{Results and discussion}

\subsection{Characterization of CNTs}

The elemental composition, surface area and pore distribution characteristics of the CNTs used in this study are detailed in Table 1. Each CNT type differed distinctly in several aspects. First, the total carbon and elemental carbon contents were significantly higher in MWNTs than in SWNTs, which indicated that the MWNTs were a higher purity than the SWNTs. The major miscellaneous element in the SWNTs was oxygen, but the hydrogen and nitrogen contents of both CNT types were similar and low. The SWNTs could be assumed to bear more oxygen-containing functional groups, such as $-\mathrm{C}-\mathrm{OO}-$ or $-\mathrm{C}=\mathrm{O}$.

Second, the diameter of SWNTs $(<2 \mathrm{~nm})$ was much smaller than that of MWNTs (20-40 nm). Third, the surface area of the SWNTs was about eight times higher than that of MWNTs, which could be attributed to the much smaller diameter and the higher micropore and mesopore (plus macropore) volumes of the SWNTs.

Table 1 Elemental composition, surface area, and pore distribution of the CNTs

\begin{tabular}{|c|c|c|c|c|c|c|c|c|}
\hline \multirow[t]{2}{*}{ CNTs } & \multirow[t]{2}{*}{$\mathrm{TC}(\%)$} & \multirow[t]{2}{*}{$\mathrm{EC}(\%)$} & \multirow[t]{2}{*}{$\mathrm{H}(\%)$} & \multirow[t]{2}{*}{$\mathrm{N}(\%)$} & \multirow[t]{2}{*}{$\mathrm{O}(\%)$} & \multirow{2}{*}{$\begin{array}{l}\text { Surface } \\
\text { area }\left(\mathrm{m}^{2} / \mathrm{g}\right)\end{array}$} & \multicolumn{2}{|c|}{ Pore volume $\left(\mathrm{cm}^{3} / \mathrm{g}\right)$} \\
\hline & & & & & & & Micro & $\mathrm{Mes}+\mathrm{Mac}$ \\
\hline SWNTs & 92.32 & 76.36 & 0.76 & 0.36 & 6.56 & 405.95 & 0.16 & 1.11 \\
\hline MWNTs & 99.98 & 97.53 & 0.92 & 0.34 & 0.01 & 51.69 & 0.017 & 0.16 \\
\hline
\end{tabular}

MWNTs: multi-walled carbon nanotubes; SWNTs: single-walled carbon nanotubes; TC: total carbon; EC: elemental carbon; Micro: micropore volume; Mes + Mac: sum of mesopore and macropore volumes. The sum of the elemental composition of MWNTs is slightly greater than $100 \%$ due to the experimental/analytical variance. 
Table 2 Elemental composition of peat humic acid (PHA) and soil humic acid (SHA)

\begin{tabular}{|c|c|c|c|c|c|c|c|c|c|}
\hline Humic acid & $\mathrm{C}(\%)$ & $\mathrm{H}(\%)$ & $\mathrm{N}(\%)$ & $\operatorname{Ash}(\%)$ & $\mathrm{O}(\%)$ & $\mathrm{H} / \mathrm{C}$ & $\mathrm{O} / \mathrm{C}$ & $\mathrm{C} / \mathrm{N}$ & $\overline{(\mathrm{N}+\mathrm{O}) / \mathrm{C}}$ \\
\hline PHA & 57.48 & 3.61 & 1.61 & 8.25 & 29.06 & 0.75 & 0.38 & 41.70 & 0.40 \\
\hline SHA & 33.15 & 4.43 & 3.38 & 31.60 & 27.44 & 1.60 & 0.62 & 11.43 & 0.71 \\
\hline
\end{tabular}

\subsection{Characterization of humic acids}

Table 2 presents the elemental analysis for the two humic acids and some calculated elemental ratios of $\mathrm{H}, \mathrm{C}$ and $\mathrm{O}$. The carbon content of PHA was greater than that of SHA and the $\mathrm{H} / \mathrm{C}$ ratio of PHA was much lower than that of SHA, which implied a higher aromaticity for PHA than SHA. Both the $\mathrm{O} / \mathrm{C}$ and $(\mathrm{N}+\mathrm{O}) / \mathrm{C}$ ratios were greater for SHA than for PHA, which indicated that SHA was more aliphatic than PHA. ${ }^{1} \mathrm{H}$ NMR spectra of the two humic acids were used to evaluate the hydrophobicity differences between SHA and PHA (Fig. 1). The peaks at 0-3.0 ppm, 3.0-6.5 ppm, 6.5-8.5 ppm were attributed to the aliphatic (apolar), polar and aromatic (apolar) hydrogens, respectively. The hydrophobic coefficients, defined as the ratio of total apolar hydrogen to polar hydrogen, were 0.71 for SHA and 0.19 for PHA. SHA had a stronger affinity for non-aromatic organic pollutants than did PHA, based on this analysis.

\subsection{Dispersion stability of CNTs after modification}

The dispersion phenomena of the MWNTs and SWNTs were distinctly different. After $1 \mathrm{~min}$ of ultrasonic agitation, the surfactant or humic acid treated SWNTs would both settle rapidly to the bottom of the glass tubes, indicating that no stable dispersion had formed. In contrast, effective dispersion was observed for MWNTs modified with either surfactant or humic acid. Figure 2 shows the absorbance changes for dispersed MWNT suspensions (diluted for absorbance measurement) with time after $1 \mathrm{~min}$ ultrasonic agitation. No appreciable absorbance decrease was observed after $24 \mathrm{hr}$ of quiescent settling, which indicated that MWNTs could be stabilized by both humic acids and surfactants.

The differences in dispersion behavior between SWNTs

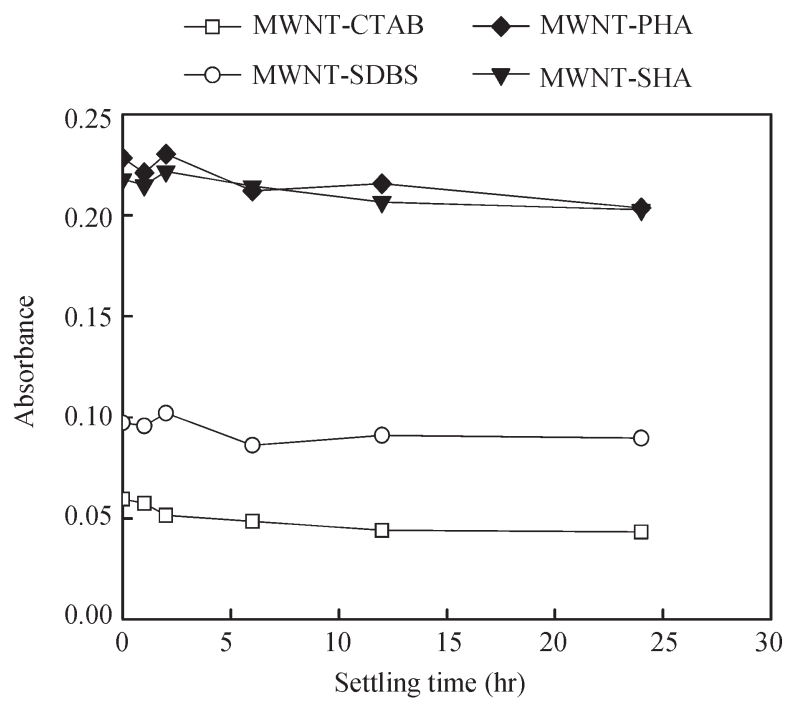

Fig. 2 Dispersion stability of humic acid and surfactant modified MWNTs. (SDBS: sodium dodecylbenzene sulfonate; CTAB: cetyltrimethylammooium bromide).

and MWNTs might be due to differences in their length/diameter aspect ratios. Compared with the diameter of MWNTs, a much smaller diameter of the SWNTs makes the tubes more flexible and easier to become entangled with each other and therefore more difficult to separate. The larger diameter of the MWNTs may prevent this type of entanglement. After humic acid and surfactant modification, the original hydrophobic surface of MWNTs was partly covered by surfactant or humic acid molecules, which increased the dispersal of the modified MWNTs in water.

TEM images of dispersed MWNTs are shown in Fig. 3. Although some MWNTs are still entangled together, single MWNTs were found in the dispersed suspensions.
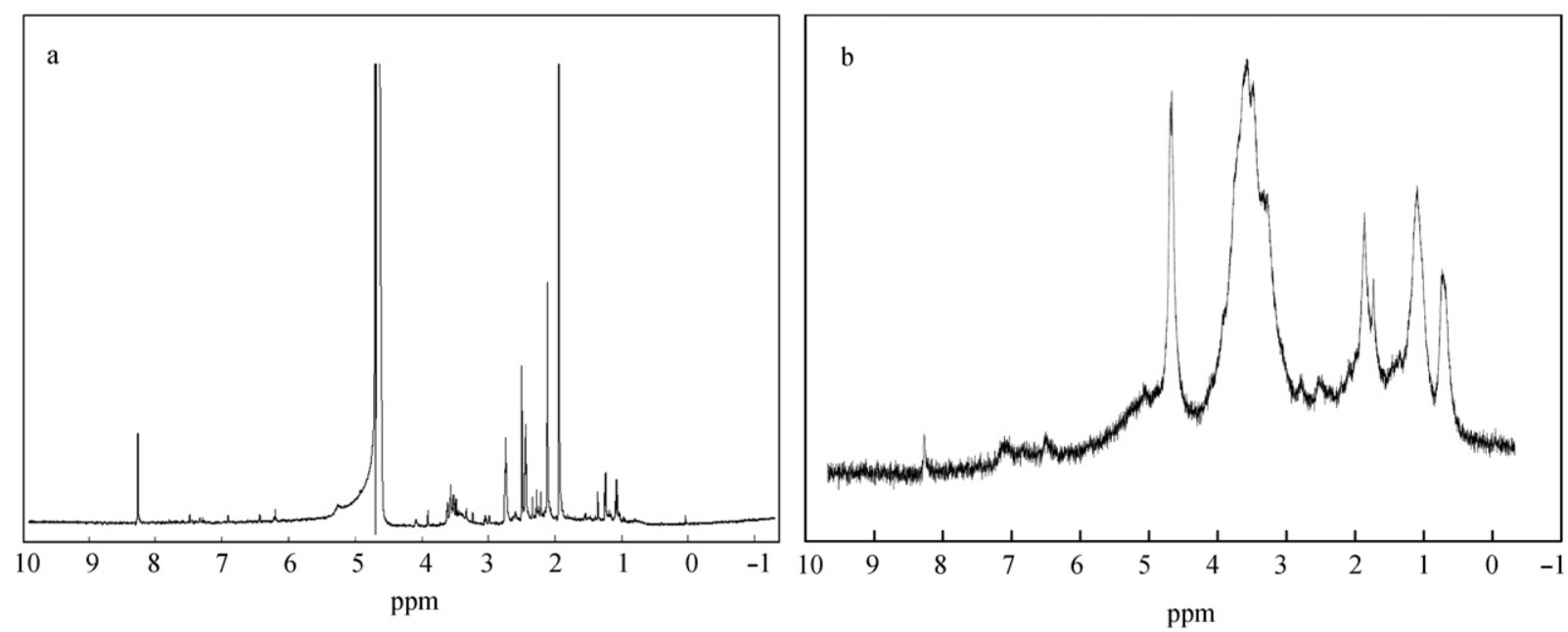

Fig. $1{ }^{1} \mathrm{H}$ NMR spectra of PHA (a) and SHA (b). 

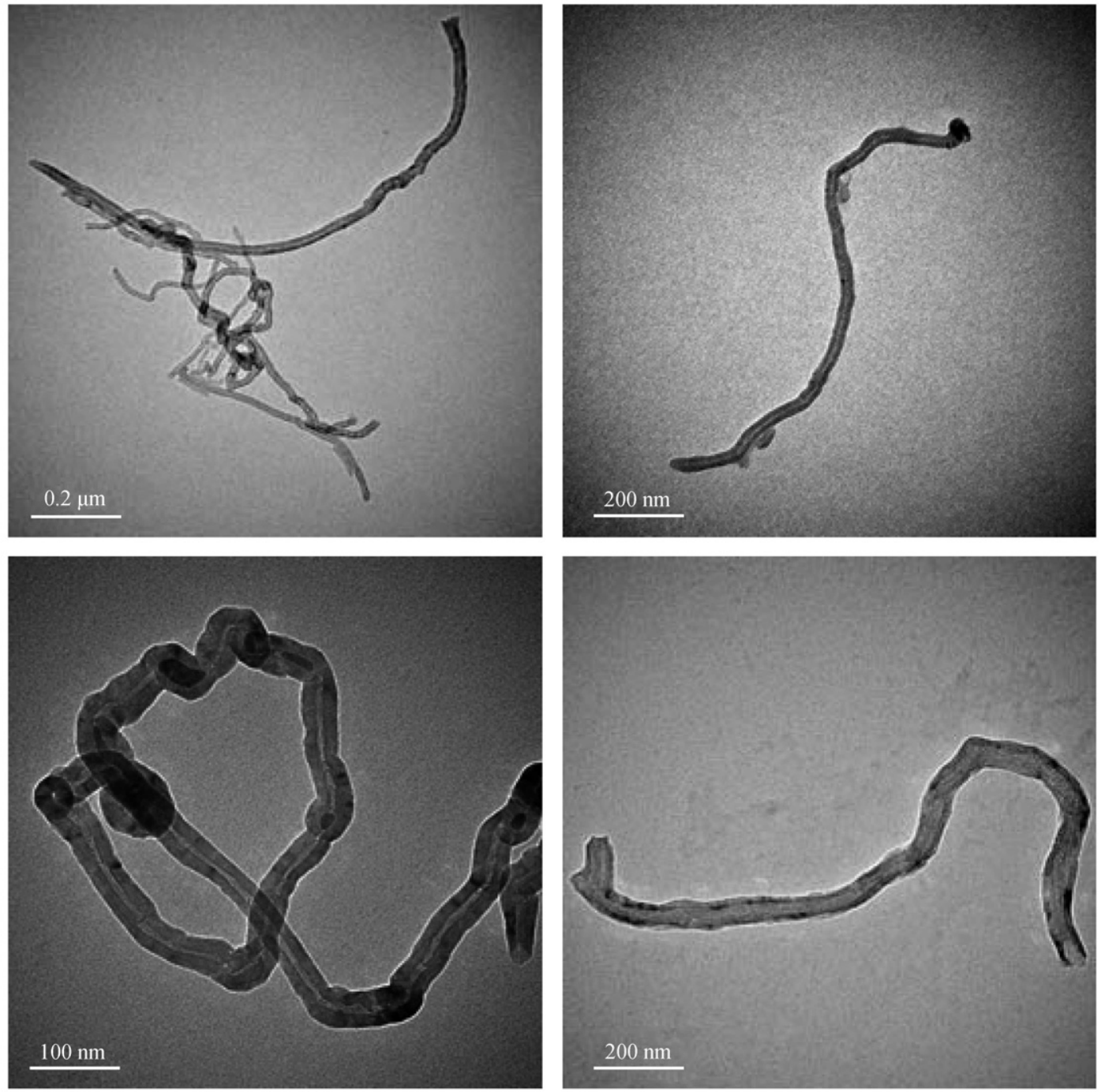

Fig. 3 Typical TEM images of MWNTs dispersed with humic acid.

\subsection{Adsorption of atrazine by humic acid dispersed CNTs}

The adsorption of atrazine by humic acid dispersed MWNTs and SWNTs is shown in Fig. 4. For comparison, the adsorption of atrazine by untreated CNTs is also presented. The adsorption of atrazine by humic acid dispersed CNTs was clearly lower than that of non-dispersed CNTs, which indicated that the pre-adsorption of humic acid could significantly inhibit the adsorption of atrazine by CNTs. All of the data were fitted to the Freundlich equation, and the fitting parameters are listed in Table 3.

In order to explain the major inhibitory effect of humic acid, surface area and micropore volume of MWNT samples after PHA modification were determined. After PHA modification, MWNT surface area was reduced from 51.69 to $48.01 \mathrm{~m}^{2} / \mathrm{g}$ and micropore volume was reduced from 0.017 to $0.014 \mathrm{~cm}^{3} / \mathrm{g}$. The reduction in surface area alone could not account for the decreased adsorption capacity. It is possible that humic acid blocked some of the micropores within the CNT bundles and thus reduced the effective surface area to some extent (the micropore volume of MWNTs decreased nearly $18 \%$ after PHA treatment). On the other hand, humic acid can significantly increase the dispersion of MWNTs, as previously discussed. Dispersion is a factor that increases the number of adsorption sites on MWNTs, due to exposure of new surfaces. Therefore, the slight surface area changes observed in humic acid treated MWNTs were the result of the balance of these two different effects of humic acid.

Since the surface area reduction and pore-blockage

Table 3 Freundlich model fitting parameters of atrazine adsorption isotherm data

\begin{tabular}{|c|c|c|c|c|c|c|c|c|c|c|}
\hline & \multirow[t]{2}{*}{ MWNTs } & \multicolumn{4}{|c|}{ Modified MWNTs } & \multirow[t]{2}{*}{ SWNTs } & \multicolumn{4}{|c|}{ Modified SWNTs } \\
\hline & & SHA & PHA & CTAB & SDBS & & SHA & PHA & CTAB & SDBS \\
\hline$\overline{K_{\mathrm{f}}}$ & 9.42 & 0.65 & 0.18 & 0.26 & 0.32 & 53.77 & 25.99 & 6.31 & 12.20 & 8.14 \\
\hline$n$ & 0.32 & 0.64 & 0.89 & 0.72 & 0.66 & 0.35 & 0.44 & 0.84 & 0.52 & 0.55 \\
\hline$R^{2}$ & 0.960 & 0.928 & 0.919 & 0.895 & 0.847 & 0.983 & 0.984 & 0.949 & 0.907 & 0.981 \\
\hline
\end{tabular}



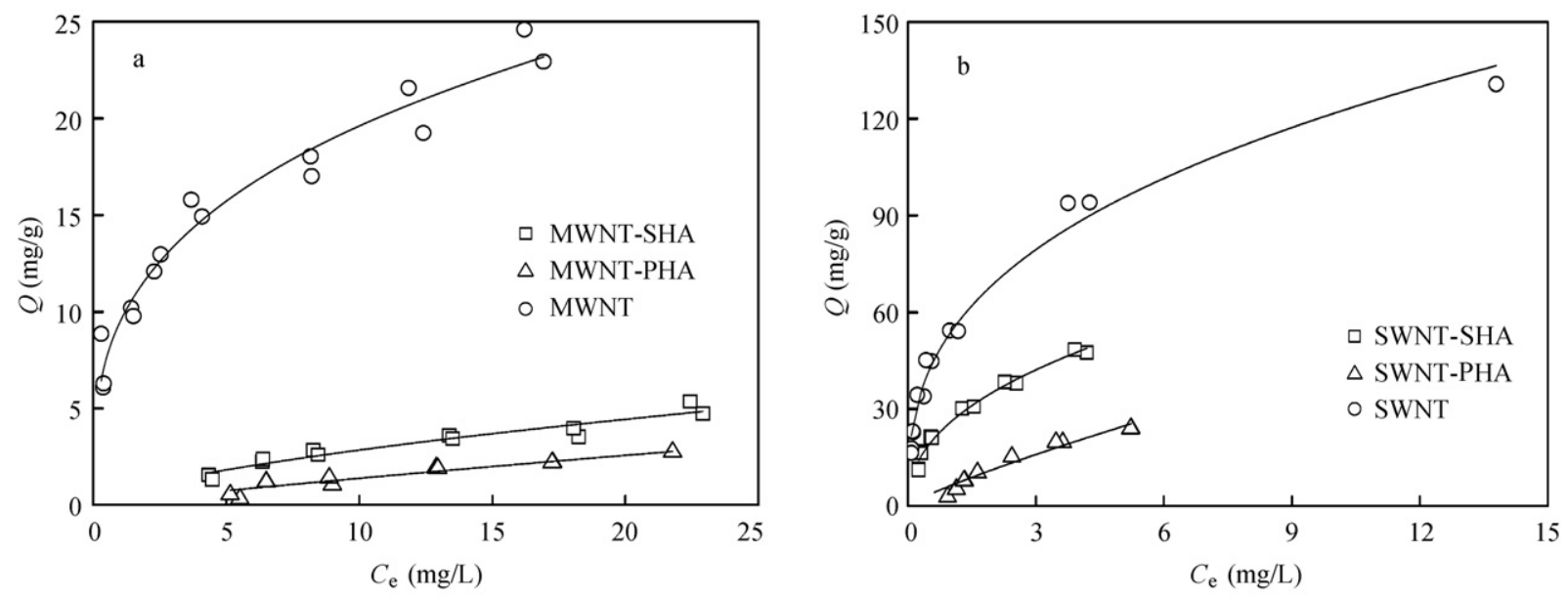

Fig. 4 Adsorption of atrazine by MWNTs (a) and SWNTs (b) before and after humic acid treatment.

effects were not the dominant factor for the inhibition of atrazine adsorption, an actual change in the surface properties induced by humic acids could account for this inhibition effect. One possible explanation is that the hydrophobicity of CNT surfaces covered by humic acids was significantly decreased, which reduced the surface affinity for atrazine.

The adsorption isotherms also showed that the atrazine adsorption onto PHA dispersed CNTs was relatively lower than that onto SHA dispersed CNTs. The humic acid dispersed CNTs had two types of adsorption sites for atrazine; humic acid covered surface sites and non-covered surface sites. Several studies have reported that the adsorption capacity of CNTs is positively correlated with the aromatic ring numbers of the adsorbates (including NOM), and the $\pi-\pi$ electron donor-acceptor interaction is an important mechanism for the adsorption of aromatic ring-containing chemicals onto CNTs (Lin and Xing, 2008; Hyung and Kim, 2008). Therefore, compared with SHA, more PHA could be adsorbed onto CNTs during the surface modification process due to its higher aromaticity, which would leave fewer exposed CNT surface sites for atrazine adsorption. For those surface areas covered by humic acids, the SHA-covered surface may have relatively higher affinity for atrazine than do PHA-covered surfaces, due to the different properties of these two humic acids.

The differences in atrazine adsorption between MWNTs and SWNTs is mainly due to their different surface areas, and micropore and mesopore (or plus macropore) volumes. The surface area and micropore volume of SWNTs are one magnitude higher than those of MWNTs, and correspondingly the amount of atrazine adsorbed onto SWNTs is nearly one magnitude higher under similar atrazine equilibrium conditions. Pan et al. (2008) also found that surface area and pore volume distribution of CNTs are major factors controlling the adsorption of polycyclic aromatic hydrocarbons and some endocrine disruptor compounds to carbon nanomaterials. Even after humic acid modification, the modified SWNTs still had a higher atrazine adsorption than humic acid modified MWNTs.

\subsection{Adsorption of atrazine by surfactant dispersed CNTs}

Figure 5 shows the adsorption of atrazine by two surfactant dispersed CNTs. Similar to what was seen following humic acid modification, surfactant treatment also significantly inhibited the atrazine adsorption by CNTs. The surfactant concentration used to disperse the CNTs was 1.5
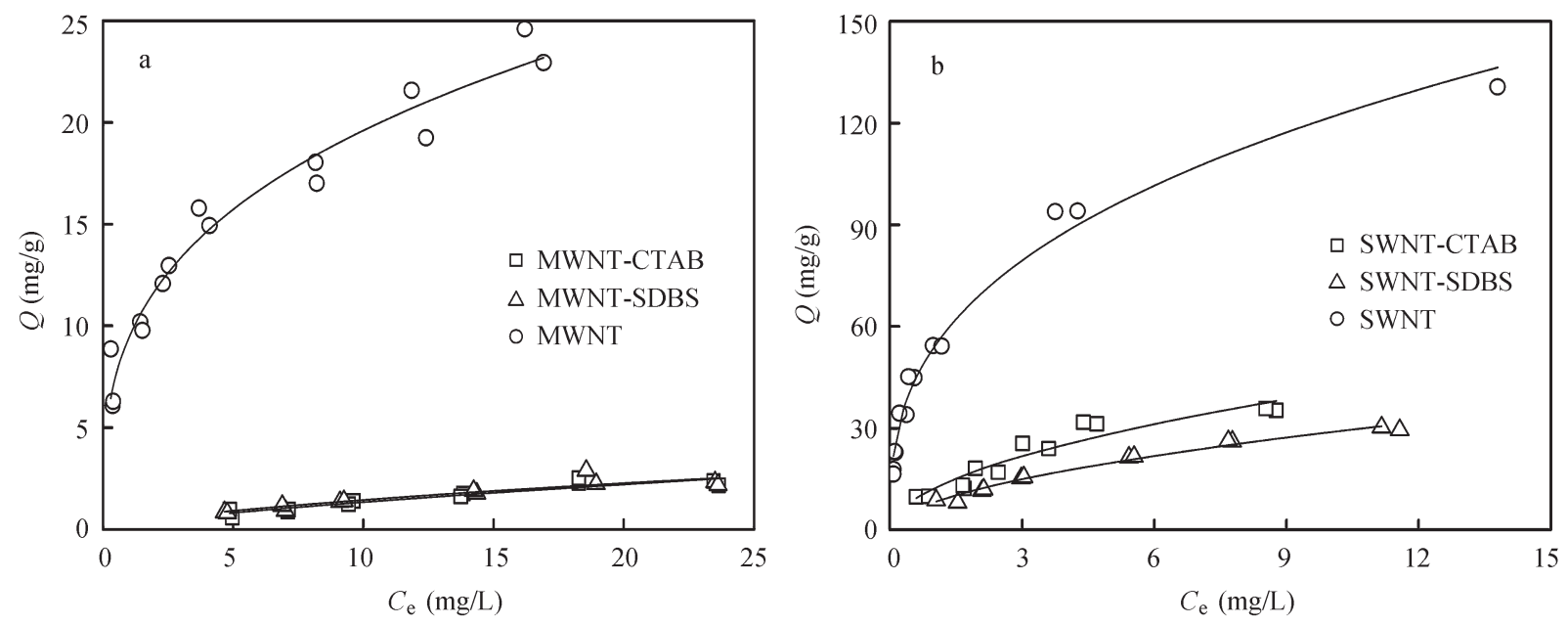

Fig. 5 Adsorption isotherms of MWNTs (a) and SWNTs (b) before and after surfactant treatment. 

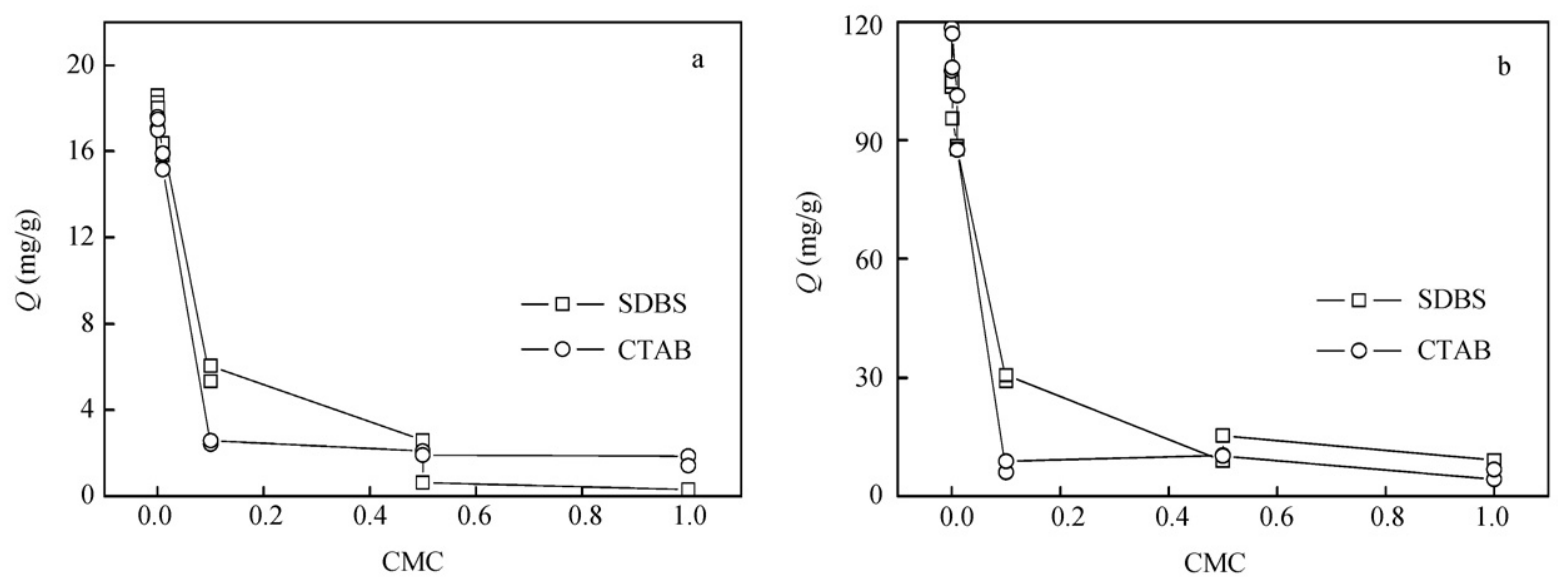

Fig. 6 Effect of surfactant concentration in solution on the adsorption of atrazine by MWNTs (a) and SWNTs (b).

CMC, therefore a portion of the surface of the CNTs may have been covered by surfactant micelles after treatment. Since the hydrophilic fraction of the surfactant micelles faces the water, the modified CNTs may have become more hydrophilic, thus reducing the adsorption of atrazine to a great extent. The inhibitory effects of cationic and anionic surfactants on the adsorption of MWNTs were similar, but the inhibitory effect of SDBS on SWNTs was somewhat greater than that of CTAB. As shown in Table 1, SWNTs were less pure and contained more oxygen atoms; the existence of oxygen containing functional groups may therefore influence the affinity of cationic and anionic surfactants, and give rise to the slight difference in inhibition of atrazine adsorption as observed. In the case of MWNTs, the high hydrophobicity of their surfaces created an equal affinity for cationic or anionic surfactants, and consequently the inhibitory effects on atrazine adsorption were similar for either surfactant.

The surfactant treatment increased the dispersion of MWNTs, and consequently an adsorption surface area increase was expected. However, this possible surface area increase did not appear to influence atrazine adsorption by the MWNTs.

\subsection{Competitive adsorption of atrazine and surfactant on CNTs}

Figure 6 shows the effect of surfactant concentration on the adsorption of atrazine by the two CNT types. With the increase in surfactant concentration, the adsorption of atrazine decreased rapidly. When the surfactant concentration was greater than $0.1 \mathrm{CMC}$, the atrazine adsorption decrease was much slower and eventually reached a constant level. The effects of the two surfactants were similar. These results indicate that a competitive adsorption effect exists between the surfactant and atrazine. When a higher surfactant concentration was used, more CNT adsorption sites were occupied by surfactants, and thus, fewer sites were available for atrazine. On the other hand, atrazine could combine with the hydrophobic portion of surfactant molecules in solution, and thus the aqueous activity of atrazine was decreased. The ionic type of surfactants exhibited little effect on the adsorption of atrazine. When the surfactant concentration was less than $0.5 \mathrm{CMC}$, the inhibitory effect of CTAB was slightly greater than that of SDBS. When the surfactant concentration was greater than $0.5 \mathrm{CMC}$, the two surfactants exhibited similar inhibitory effects on the atrazine adsorption.

\section{Conclusions}

This study examined the effects of surface modification on the dispersion of CNTs and on their capacity to adsorb an organic pollutant, atrazine. Humic acids with different characteristics and surfactants with different ionic properties effectively dispersed MWNTs, but could not significantly disperse SWNTs under the conditions used in this study. Both humic acid and surfactant treatments greatly repressed the adsorption of atrazine by CNTs. Humic acid with higher aromaticity exhibited a higher inhibitory effect on atrazine adsorption. No great difference in inhibition effect was noted in term of the ionic character of the surfactants. Competitive adsorption of surfactant and atrazine onto the CNTs was observed.

The adsorption of organic pollutants by untreated or surface modified nanomaterials involves complex mechanisms. Detailed properties of nanomaterials, surface modification reagents, the degree of dispersion, the characteristics of organic pollutants, and the interactions between each, need to be considered. With the coming of the nanotechnology era, greater attention should be given to the environmental behaviors of the CNTs, either as emerging contaminants themselves or as carriers of bound organic pollutants.

\section{Acknowledgments}

This research was supported by the National Natural Science Foundation of China (No. 20777081, 50878204, 20537020) and the Scientific Research Foundation for the Returned Overseas Chinese Scholars, State Education Ministry of China.

\section{References}

Chen W, Duan L, Zhu D Q, 2007. Adsorption of polar and nonpolar organic chemicals to carbon nanotubes. Environmental Science and Technology, 41: 8295-8300. 
Chingombe P, Saha B, Wakeman R J, 2006. Sorption of atrazine on conventional and surface modified activated carbons. Journal of Colloid and Interface Science, 302: 408-416.

Colvin V L, 2003. The potential environmental impact of engineered nanomaterials. Nature Biotechnology, 21: 11661170.

Hyung H, Frotner J D, Hughes J B, Kim J H, 2007. Natural organic matter stabilizes carbon nanotubes in the aqueous phase. Environmental Science and Technology, 41: 179184.

Hyung H, Kim J H, 2008. Natural organic matter (NOM) adsorption to multi-walled carbon nanotubes: effect of NOM characteristics and water quality parameters. Environmental Science and Technology, 42: 4416-4421.

Jia G, Wang H F, Yan L, Wang X, Pei R J, Yan T et al., 2005. Cytotoxicity of carbon nanomaterials: single-wall nanotube, multi-wall nanotube, and fullerene. Environmental Science and Technology, 39: 1378-1383.

Jiang L Q, Gao J, Sun J, 2003. Production of aqueous colloidal dispersions of carbon nanotubes. Journal of Colloid and Interface Science, 260: 89-94.

Kuo C Y, Wu C H, Wu J Y, 2008. Adsorption of direct dyes from aqueous solutions by carbon nanotubes: Determination of equilibrium, kinetics and thermodynamics parameters. Journal of Colloid and Interface Science, 327: 308-315.

Li Q, Snoeyink V L, Campos C, Marin B J, 2002. Displacement effect of NOM on atrazine adsorption by PACs with different pore size distributions. Environmental Science and Technology, 36: 1510-1515.

Lin D H, Xing B S, 2008. Adsorption of phenolic compounds by carbon nanotubes: role of aromaticity and substitution of hydroxyl groups. Environmental Science and Technology, 42: 7254-7259.

Lisunova M O, Lebovka N I, Melezhyk O V, Boiko Y P, 2006. Stablilty of the aqueous suspensions of nanotubes in the presence of nonionic surfactant. Journal of Colloid and Interface Science, 299: 740-746.

Lu J J, Li Y, Yan X M, Shi B Y, Wang D S, Tang H X, 2009. Sorption of atrazine onto humic acids (HAs) coated nanoparticles. Colloids and Surfaces A: Physicochemical Engineering Aspects, 347: 90-96.

Magrez A, Kasas S, Salicio V, Pasquier N, Seo J W, Celio M et al., 2006. Celluar toxicity of carbon-based nanomaterials. Nano Letters, 6: 1121-1125.

Marsh D H, Rance G A, Zaka M H, Whitby R J, Khlobystov A N, 2007. Comparison of the stability of multiwalled carbon nanotube dispersions in water. Physical Chemistry Chemical Physics, 9: 5490-5496.

Mauter M S, Elimelech M, 2008. Environmental applications of carbon-based nanomaterials. Environmental Science and Technology, 42: 5843-5859.
Monteiro-Riviere N A, Inman A O, 2006. Challenges for assessing carbon nanomaterial toxicity to the skin. Carbon, 44: 1070-1078.

Murphy E M, Zachara J M, Smith S C, Philips J L, 1992. The sorption of humic acids to mineral surfaces and their role in contaminant binding. Science of the Total Environment, 117-118: 413-423.

Pan B, Lin D, Mashayekhi H, Xing B, 2008. Adsorption and hysteresis of bisphenol A and 17 -ethinyl estradiol on carbon nanomaterials. Environmental Science and Technology, 42: $5480-5485$.

Pan B, Xing B S, 2008. Adsorption mechanisms of organic chemicals on carbon nanotubes. Environmental Science and Technology, 42: 9005-9013.

Peng X J, Li Y H, Luan Z K, Di Z C, Wang H Y, Tian B H et al., 2003. Adsorption of 1,2-dichlorobenzene from water to carbon nanotubes. Chemical Physics Letters, 376(1-2): $154-158$.

Robichaud C O, Tanzil D, Weilenmann U, Wiesner M R, 2005. Relative risk analysis of several manufactured nanomaterials: an insurance industry context. Envrionmetal Science and Technology, 39: 8985-8994.

Salloum M J, Dudas M J, McGill W B, 2001. Variation of 1naphthol sorption with organic matter fractionation: the role of physical conformation. Organic Geochemistry, 32: 709719.

Vaisman L, Wagner H D, Marom G, 2006. The role of surfactants in dispersion of carbon nanotubes. Advances in Colloid and Interface Science, 128-130: 37-46.

Wang X L, Lu J L, Xing B S, 2008. Sorption of organic contaminants by carbon nanotubes: influence of adsorbed organic matter. Environmental Science and Technology, 42: 3207-3212.

Yan X M, Shi B Y, Lu J J, Feng C H, Wang D S, Tang H X, 2008. Adsorption and desorption of atrazine on carbon nanotubes. Journal of Colloid and Interface Science, 321: 30-38.

Yang K, Wang X L, Zhu L Z, Xing B S, 2006. Competitive sorption of pyrene, phenanthrene, and naphthalene on multiwalled carbon nanotubes. Environmental Science and Technology, 40: 5804-5810.

Yang K, Zhu L Z, Xing B S, 2006. Adsorption of polycyclic aromatic hydrocarbons by carbon nanomaterials. Environmental Science and Technology, 40: 1855-1861.

Yu J, Grossiord N, Koning C E, Loos J, 2007. Controlling the dispersion of muti-wall carbon nanotubes in aqueous surfactant solution. Carbon, 45: 618-623.

Zhuang X Y, Shi B Y, Lu J J, Yan X M, Gao Y F, 2009. Adsorption and desorption behavior of atrazine on carbon nanotubes. Acta Scientiae Circumstantiae, 29(6): 12451251. 